\title{
Radiographic Progression of Degenerative Lumbar Scoliosis after Short Segment Decompression and Fusion
}

\author{
Dae-Woo Hwang*, Suk-Ha Jeon*, Ju-Wan Kim*, \\ Eung-Ha Kim ${ }^{\dagger}$, Jung-Hee Lee ${ }^{\ddagger}$, Kyoung-Jun Park ${ }^{\ddagger}$ \\ *Department of Orthopedic Surgery, National Medical Center, Seoul, Korea \\ ${ }^{\dagger}$ Department of Orthopedic Surgery, Soonchunhyang University College of Medicine, Bucheon, Korea \\ ${ }^{\ddagger}$ Department of Orthopedic Surgery, Kyung Hee University College of Medicine, Seoul, Korea
}

Study Design: A retrospective study

Purpose: To assess the radiographic progression of degenerative lumbar scoliosis after short segment decompression and fusion without deformity correction.

Overview of Literature: The aims of surgery in degenerative lumbar scoliosis are the relief of low back and leg pain along with a correction of the deformity. Short segment decompression and fusion can be performed to decrease the level of low back and leg pain provided the patient is not indicated for a deformity correction due to medical problems. In such circumstance, the patients and surgeon should be concerned with whether the scoliotic angle increases postoperatively.

Methods: Forty-seven patients who had undergone short segment decompression and fusion were evaluated. The average follow-up period was more than 3 years. The preoperative scoliotic angle and number of fusion segments was $13.6 \pm 3.9^{\circ}$ and $2.3 \pm 0.5$, respectively. The preoperative, postoperative and last follow-up scoliotic angles were compared and the time of progression of scoliotic angle was determined.

Results: The postoperative and last follow-up scoliotic angle was $10.4 \pm 2.3^{\circ}$ and $12.1 \pm 3.6^{\circ}$, respectively. In eight patients, conversion to long segment fusion was required due to the rapid progression of the scoliotic angle that accelerated from 6 to 9 months after the primary surgery. The postoperative scoliosis aggravated rapidly when the preoperative scoliotic angle was larger and the fusion was extended to the apical vertebra.

Conclusions: The scoliotic angle after short segment decompression and fusion was not deteriorated seriously in degenerative lumbar scoliosis. A larger scoliotic angle and fusion to the apical vertebra are significant risk factors for the acceleration of degenerative lumbar scoliosis.

Key Words: Degenerative lumbar scoliosis, Short segment fusion, Radiographic progression

\section{Introduction}

Degenerative lumbar scoliosis is one of the most common causes of low back and leg pain in the aged population. Vanderpool et al. ${ }^{1}$, in the early stage of their active research on degenerative lumbar scoliosis, reported that asymmetric compression of the vertebrae by metabolic bone diseases, such as osteoporosis and osteomalacia, were the causes of scoliosis. However, since it has been disclosed that there is no difference in the bony quality of adults that had suffered from adolescent idiopathic scoliosis, the etiological theory on degenerative lumbar scoliosis has been highlighted again, and degeneration of the intervertebral disc and

Received Oct 28, 2009; 1st revised Nov 3, 2009; accepted Nov 3, 2009

Corresponding author: Kyoung-Jun Park, MD

Department of Orthopedic Surgery, Kyung Hee University College of Medicine

149 Sangil-dong, Gangdong-gu, Seoul 134-727, Korea

Tel: +82-2-440-6152, Fax: +82-2-440-7454, E-mail: discectomy@empal.com 
arthrosis of facet joint have been publicized as the outset of such manifestations ${ }^{2-4}$.

Degenerative lumbar scoliosis is a unique disease entity in the aged population, and usually manifests as osteoporosis and a variety of medical co-morbidities. Therefore, the surgical outcome is not always promising, in that many surgeons manage this disease in a conservative manner. However, in degenerative lumbar scoliosis, a wide range of neurological symptoms manifest through the supervening of spondylolisthesis, lateral listhesis, rotary subluxation and spinal stenosis along with an imbalance in the coronal and sagittal plane of the vertebrae. Therefore, surgeons must make a decision as to whether to perform surgery.

Therefore, the aim of surgical treatment is to reduce lower back and radiating pain in the lower extremities along with a correction of the deformity. That is, a deformity correction and long segment fusion for the correction of an imbalance in the coronal and sagittal plane of the vertebrae are necessary, and include decompression of the corresponding regions in accordance with the extent of neural compression $^{5.6}$.

Before surgery, the state of health and daily lifestyle of the patient must be considered sufficiently in conjunction with the main symptoms and radiological abnormal findings of the patients. If long segment fusion is not possible or recommended in accordance with the overall state of the patient, then short segment decompression and fusion should be executed in a limited manner by focusing on a decrease in low back pain and leg pain. However, in such cases, the change in scoliotic curvature will accelerate after short segmentation decompression and fusion ${ }^{5.7}$. Accordingly, this study examined the changes in the scoliotic curvature retrospectively and deduced the factors that accelerate curvature in cases in whom only short segment decompression and fusion were carried out without a deformity correction for degenerative lumbar scoliosis.

\section{Materials and Methods}

Among the patients with degenerative lumbar scoliosis with a Cobb's angle $>10^{\circ}$ in the whole spine standing posteroanterior radiograph, 117 patients, who had received short segmentation decompression and fusion that did not exceed the apical vertebra and $<3$ segments during the period from March 2004 to March 2006, were collected. Of these 117 patients, 47 patients who were observed for more than 3 years were finally selected as subjects.

The average follow-up duration was $3.4 \pm 0.6$ ( 3 to 5 ) years, and the average age of the patients was 65.9 (45 to $81)$ years. The subjects consisted of 12 males and 35 females.

The apical vertebra was L2, L3 and L4 in 5, 33 and 9 patients, respectively. The fusion level was L4-5 in 3 patients and L5-S1 in 1 patient in one segment fusion, L3-5 in 19 patients and L4-S1 in 12 patients in two segments fusion, and L1-4 in 1 patient, L2-L5 in 2 patients and L3-S1 in 9 patients with three segments fusion. The average number of levels fused was $2.3 \pm 0.5$ (Table 1).

As surgical methods, decompression of the area that induced the clinical symptoms was carried out. Fixation was performed using a pedicle screw. With regard to the fusion methods, posterolateral fusion was performed in 7 patients, while posterior lumbar interbody fusion using a cage was performed in 40 patients.

Fusion over the apical vertebra was not carried out, and the scoliotic curvature on the coronal plane was measured using the Cobb's angle on the whole spine standing posteroanterior radiographs as soon as possible after surgery (2 weeks after surgery), 3 months, 6 months and 9 months after surgery, and at last follow-up. In addition, changes in the scoliotic curvature and wedging of the upper adjacent disc of the apical vertebra were recorded and analyzed. The time for the resumption of curve progression was forecasted in this manner.

With regard to statistical analysis, a paired t-test was carried out to determine the value prior to surgery and each period using SPSS ver. 11.0 (SPSS Inc., Chicago, IL, USA).

Table 1. Summary of data

\begin{tabular}{lcr}
\hline \hline & Level & No. \\
\hline Apical vertebra & L2 & 5 \\
L3 & 33 \\
L4 & 9 \\
\hline No. of fusion segments & 1 segment & 4 \\
& L4-5 & 3 \\
& L5-S1 & 1 \\
\cline { 2 - 3 } & 2 segment & 31 \\
L3-5 & 19 \\
L4-S1 & 12 \\
\cline { 2 - 3 } & 3 segment & 12 \\
L1-4 & 1 \\
L2-5 & 2 \\
L3-S1 & 9 \\
\hline
\end{tabular}


Statistical significance was defined as $\mathrm{p}<0.05$.

\section{Results}

\section{Changes in the scoliotic curvature}

The results after an average follow-up of $3.4 \pm 0.6$ years

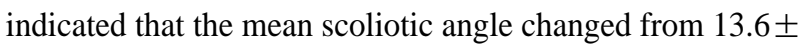
$3.9^{\circ}$, prior to surgery to $10.4 \pm 2.2^{\circ}$ as soon as possible after surgery ( 2 weeks), to $10.8 \pm 3.4^{\circ}, 10.7 \pm 3.4^{\circ}$, and $11.9 \pm$ $3.2^{\circ}$ at 3,6 , and 9 months after surgery, respectively, and $12.1^{\circ} \pm 3.6^{\circ}$ at the last follow-up. Statistical analysis of these values showed significant improvement in scoliotic curvature at all periods compared to that before surgery (as soon as possible after surgery, and 3,6, and 9 months after surgery [p<0.01], and at the last follow-up [p=0.05]). However, when comparing the value as soon as possible after surgery with that 3 and 6 months following surgery, there was no significant change. A significant loss of correction began to occur from 9 months after surgery $(\mathrm{p}<0.01)$ (Table 2, Figs. 1 and 2).

Although it is difficult to correct the scoliotic curvature through decompression and fusion of the short segment, it is possible to obtain a certain degree of curvature correction by performing the appropriate level of compression after fixation with a pedicle screw, and in posterior lumbar interbody fusion using a cage. In addition, it was possible to make the wedged disc parallel, thereby obtain a correction of curvature (Fig. 3).

\section{Changes in the disc wedging angle}

Table 2. Statistics in variation of Cobb's angle (paired t-test)

\begin{tabular}{lccc}
\hline \hline & Mean & SD & p-value \\
\hline Pre - IPO & 3.16 & 3.44 & 0.00 \\
Pre - PO3 & 2.70 & 3.53 & 0.00 \\
Pre - PO6 & 2.87 & 3.09 & 0.00 \\
Pre - PO9 & 1.63 & 3.02 & 0.01 \\
Pre - last & 1.56 & 3.59 & 0.05 \\
\hline IPO - 1PO3 & -0.47 & 2.82 & 0.26 \\
IPO - PO6 & -0.29 & 2.52 & 0.44 \\
IPO - PO9 & -1.53 & 2.53 & 0.00 \\
IPO - last & -1.60 & 2.64 & 0.00 \\
\hline
\end{tabular}

SD: standard deviation.

Pre, IPO, PO3, PO6, PO9, last: Cobb's angle at preoperative, immediately postoperative, postoperative 3-, 6-, 9-months and last follow-up period.
The wedging angle of the upper adjacent disc of apical vertebra prior to surgery was measured, and their average values changed from $1.80 \pm 1.7^{\circ}$ prior to surgery to $2.10 \pm$ $1.6^{\circ}$ as soon as possible after surgery, to $2.39 \pm 1.6^{\circ}, 2.61$ $\pm 1.5^{\circ}$, and $2.64 \pm 1.5^{\circ}$ at 3,6 , and 9 months following surgery, respectively, and to $2.86 \pm 1.8^{\circ}$ at the last followup. Statistical analysis showed a significant increase in the disc wedging angle at all periods compared to that prior to surgery, and was as that taken as soon as possible after surgery $(\mathrm{p}<0.01)$. However, there was no significant increase in the disc wedging angle after 6 months following surgery ( $>0.05)$ (Table 3$)$.

\section{Analysis on complications and revision}

Among the 47 patients, loosening of the pedicle screw was observed in 18 patients and a compression fracture of the upper adjacent vertebra occurred in 2 patients. Although nonunion was suspected in 18 patients with a loosening of the pedicle screw, revision was not decided due to nonunion or loosening of the pedicle screw.

In 8 cases, conversion to long segment fusion was performed due to the rapid progression of scoliotic curvature along with a recurrence of severe low back pain. In the case of 8 cases of revision, the mean scoliotic angle before surgery was $16.4 \pm 5.4^{\circ}$, which was higher than the overall average. The mean angle of corrected scoliotic angle after

Table 3. Statistics in variation of disc wedging (paired t-test)

\begin{tabular}{lccc}
\hline \hline & Mean & SD & p-value \\
\hline Pre - IPO & -0.31 & 1.25 & 0.104 \\
Pre - PO3 & -0.59 & 1.47 & 0.009 \\
Pre - PO6 & -0.81 & 1.38 & 0.000 \\
Pre - PO9 & -0.85 & 1.53 & 0.001 \\
Pre - last & -1.06 & 2.00 & 0.001 \\
IPO - PO3 & -0.29 & 0.92 & 0.041 \\
IPO - PO6 & -0.51 & 1.05 & 0.002 \\
IPO - PO9 & -0.54 & 1.13 & 0.002 \\
IPO - last & -0.75 & 1.50 & 0.001 \\
PO3 - PO6 & -0.22 & 0.61 & 0.017 \\
PO3 - PO9 & -0.26 & 0.78 & 0.030 \\
PO3 - last & -0.47 & 1.20 & 0.011 \\
PO6 - PO9 & -0.03 & 0.50 & 0.645 \\
PO6 - last & -0.24 & 1.13 & 0.151 \\
PO9 - last & -0.21 & 0.90 & 0.120 \\
\hline
\end{tabular}

SD: standard deviation.

pre, IPO, PO3, PO6, PO9, last: Disc wedging angle at preoperative, immediately postoperative, postoperative 3-, 6-, 9months and last follow-up period. 

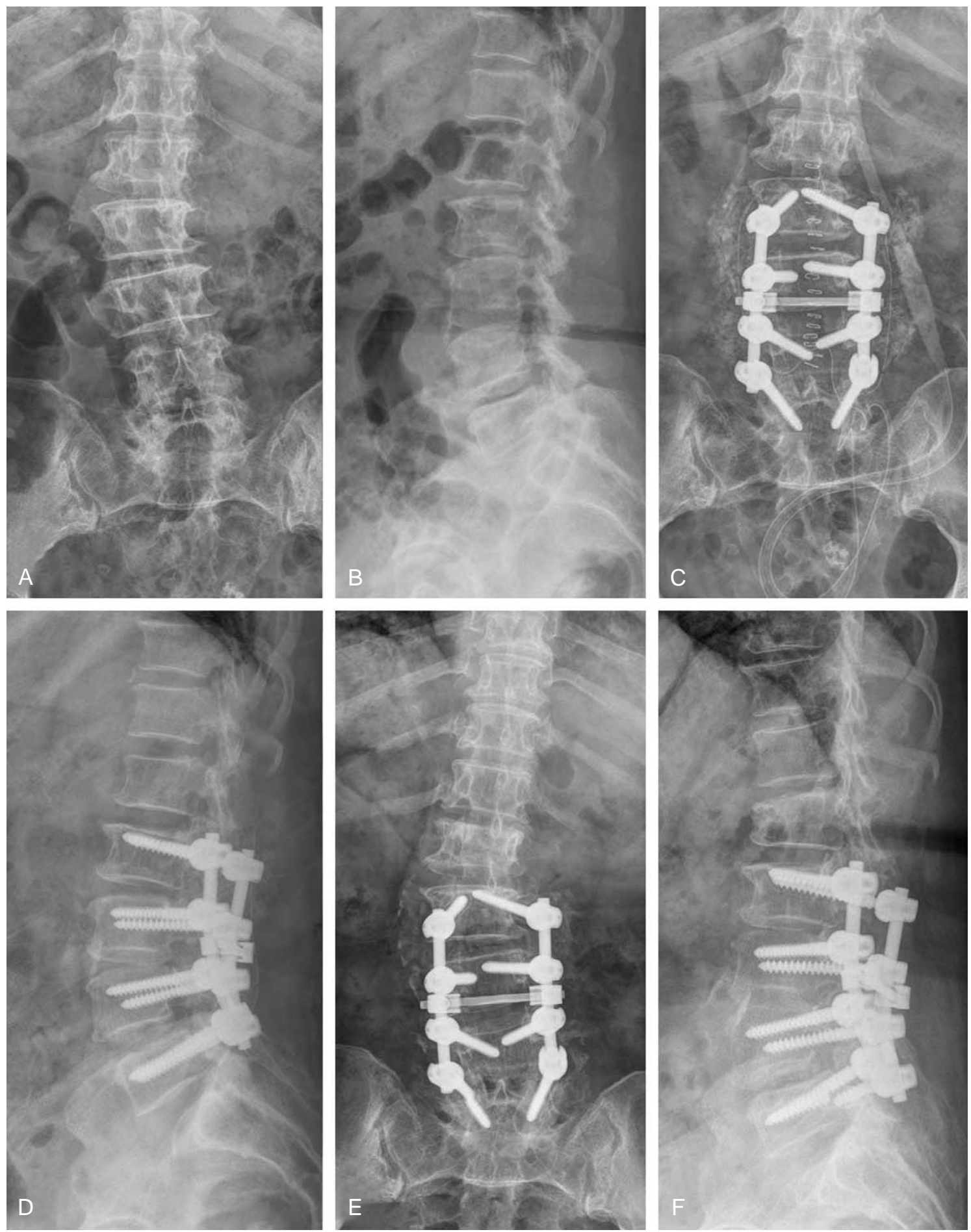

Fig. 1. (A, B) A 68 year-old female has degenerative lumbar scoliosis with a scoliotic curve and disc wedging of $15^{\circ}$ and $2.7^{\circ}$, respectively, at the L1-2 level. (C, D) Posterior decompression and posterolateral fusion were performed. The postoperative Cobb's angle and disc wedging is $11^{\circ}$ and $3.2^{\circ}$, respectively. (E, F) One year after short segment fusion, the Cobb's angle and disc wedging are increased to $16^{\circ}$ and $4.1^{\circ}$, respectively. Compression fracture of L1 and screw breakage and loosening of L5 are noted.

surgery was $12.8 \pm 2.3^{\circ}$, which is also poorer improvement compared to the overall average. The mean scoliotic angle prior to the revision was $15.4 \pm 3.6^{\circ}$. Three patients had an upper adjacent fracture, and the remainder had upper adjacent segment disease. The fusion levels were L3-5, L4-S1 and L3-S1 in 3, 4 and 1 patient, respectively. Specifically, 
there is a trend for rapid progression of the scoliotic curvature from 6 to 9 months, which is even more serious when the scoliotic angle is larger before surgery and if the upper end of the fusion corresponds to the apical vertebra in the fusion of 2-3 segments.

\section{Discussion}

Degenerative changes and facet arthrosis are the etiology of degenerative lumbar scoliosis. Kobayashi et al. ${ }^{3}$, in their study on the prevalence of degenerative lumbar scoliosis, reported that a lateral osteophyte $>5 \mathrm{~mm}$ and disc wedging $>3^{\circ}$ were risk factors for degenerative lumbar scoliosis, and found these to be the most important factors for the degenerative changes in the intervertebral disc. In addition, one must be concerned with facet arthrosis along with spondylolisthesis, lateral listhesis and rotary subluxation in establishing a treatment for degenerative lumbar scoliosis.

Surgical methods of degenerative lumbar scoliosis can be defined as decompression, deformity correction and fusion ${ }^{5,6}$. However, either all or some of these are carried out according
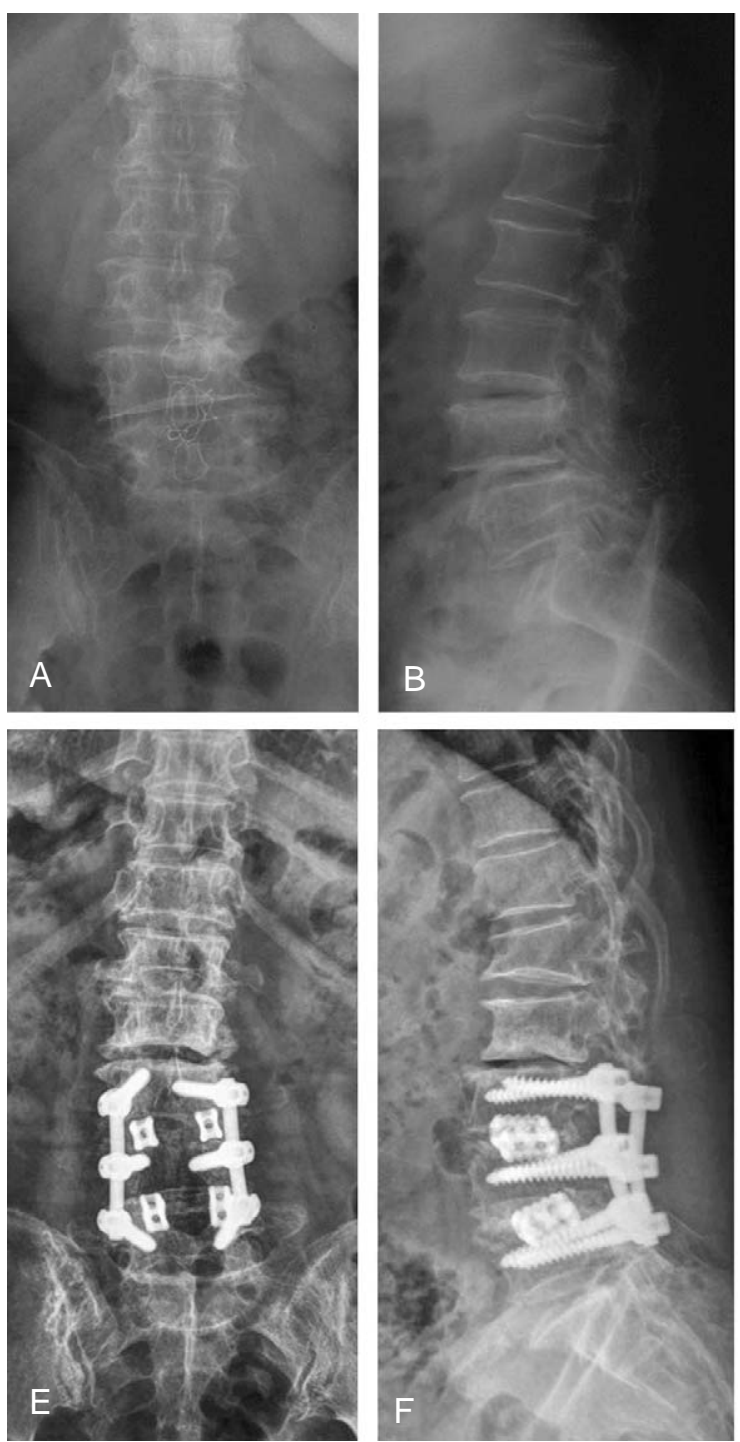
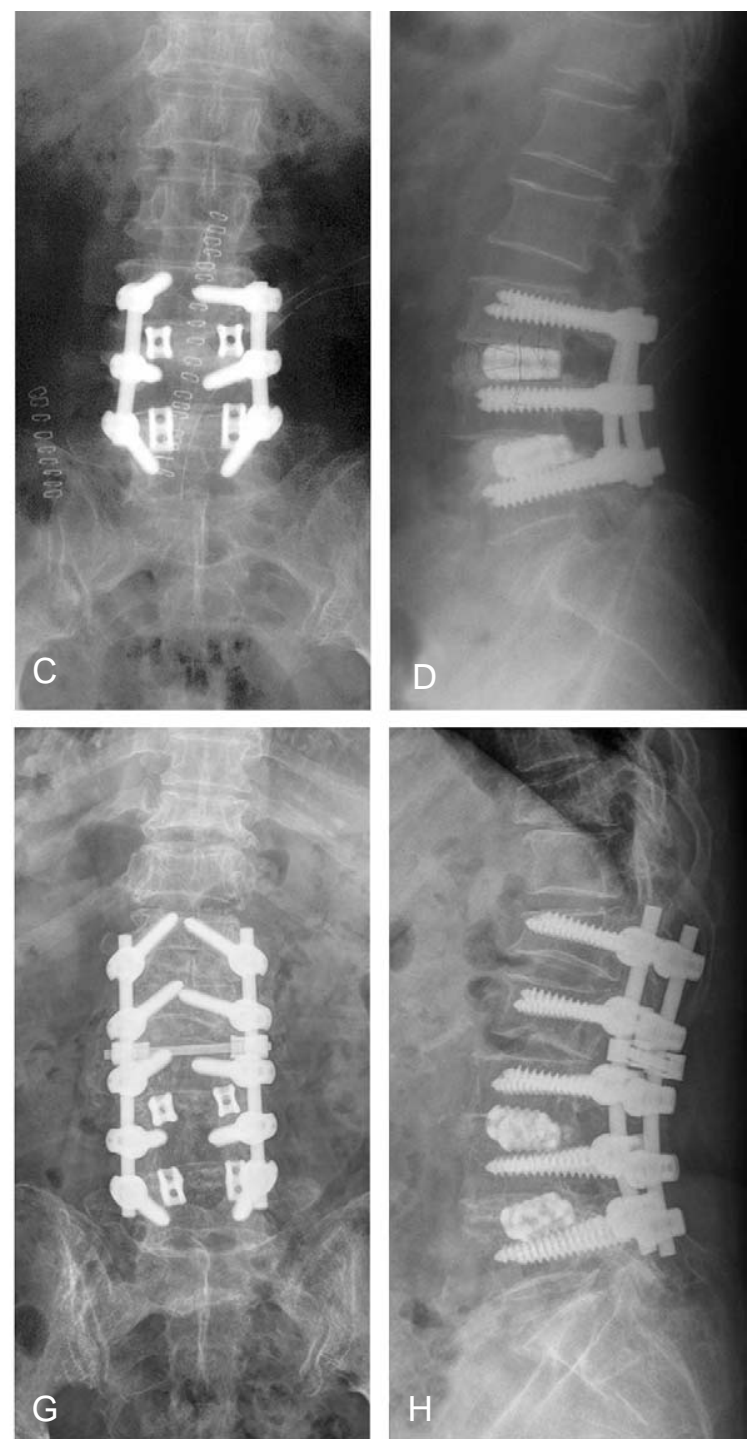

Fig. 2. (A, B) A 69 year-old female has degenerative lumbar scoliosis with a scoliotic curve and disc wedging of $15^{\circ}$ and $1.2^{\circ}$, respectively, at the L2-3 level, and degenerative spondylolisthesis L4 on L5. (C, D) Posterior decompression and posterior lumbar interbody fusion are performed. The postoperative Cobb's angle and disc wedging was $7^{\circ}$ and $1.8^{\circ}$, respectively. (E, F) Three years after short segment fusion, the upper adjacent segments shows severe degeneration with disc space narrowing and vacuum phenomenon and compression fracture of L1. The Cobb's angle and disc wedging increased to $14^{\circ}$ and $2.7^{\circ}$ respectively. $(\mathbf{G}, \mathbf{H})$ Revision up to L1 body was performed and the Cobb's angle improved to $10^{\circ}$. 

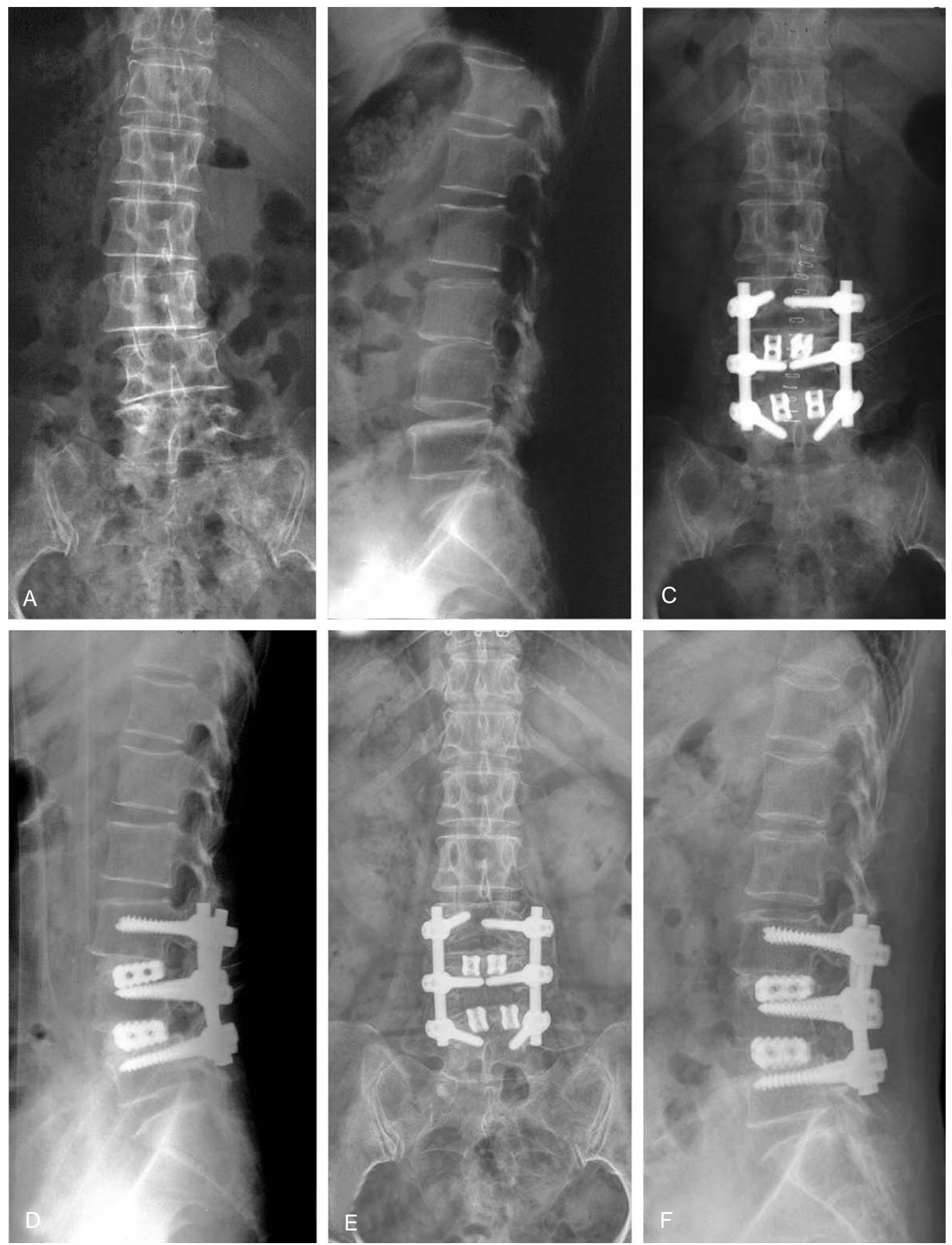

Fig. 3. (A, B) A 64 year-old female with degenerative lumbar scoliosis with a scoliotic curve and disc wedging of $13^{\circ}$ and $3.1^{\circ}$, respectivelyat the L2-3 level. (C, D) Posterior decompression, posterior lumbar interbody fusion and posterolateral fusion were performed. The postoperative Cobb's angle and disc wedging was $3^{\circ}$ and $1.1^{\circ}$, respectively. (E, F) Two years after short segment fusion, the Cobb's angle and disc wedging were not changed significantly.

to the medical condition and daily lifestyle of the patient.

Simmons ${ }^{8}$ reported that simple decompression is not recommended because it may aggravate the instability particu- larly in the apex of the curvature. However, they performed decompression at the apex of the deformity, which was approximately lateral listhesis or spondylolisthesis. This can 
be considered if it is to be applied to areas below the apical vertebrae. However, Kim et al. ${ }^{9}$ reported that the lower lumbar region is important in the occurrence of degenerative lumbar scoliosis, and its risk must always be considered to be an abnormal load always applied to the lower portion of the scoliotic curvature.

Secondly, Tribus reported that short segment decompression and fusion can be attempted in cases without coronal or sagittal imbalance ${ }^{10}$. He asserted that this method is indicated if the scoliotic curvature is small or spondylolisthesis and lateral listhesis is not severe, and that fusion should be performed for the areas that have undergone decompression. This study examined the effects of short segment decompression and fusion within 3 segments performed on the selected patients. The scoliotic angle was within $15^{\circ}$, which are relatively small angles, and there was no imbalance in the coronal and sagittal planes. Patients who require a deformity correction and long segment fusion due to the large angle were excluded. The level of correction of the scoliotic angle after surgery was $26 \%$, which is similar to the values reported by Cho et al. ${ }^{5}$. A significant increase in scoliotic curvature after surgery began from 9 months after surgery, and was more definite if the scoliotic angle was large before surgery or fusion was stopped at the apical vertebra. Therefore, in order to prevent acceleration of the scoliotic curvature, fusion must not be stopped at the segment that still has a deformity. Rather, fusion must be performed to also include the upper segments. In addition, the correlation between the symptoms of the patients and time of the acceleration of the scoliotic curvature should be considered at the follow up patient that underwent short segment fusion.

Finally, deformity correction and long segment fusion is appropriate for patients with a large Cobb angle or sagittal imbalance. This method is recommended as the principle of degenerative lumbar scoliosis treatment. However, fusion of the long segment and additional surgery for anterior support might increase the surgery time, cause significant blood loss, prolong the hospitalization time and result in detrimental medical complications, with an occurrence of 41 to $62 \%$ depending on the researchers ${ }^{8,11-13}$. Therefore, such a method is often inappropriate if the patient is old and/or in poor health, or has a lifestyle of sitting on the floor in Korea and those living in rural areas. Accordingly, the reality in Korea is that deformity correction and long segment fusion cannot be used concurrently in all patients.

Ha et al. ${ }^{14}$ whose research involved similar subjects, fol- low-up periods and fusion levels to this study stated that decompression is essential and lateral listhesis must also be fixed in order to prevent the progression of scoliosis in the proximal segment. However, the research by $\mathrm{Ha}$ et al. ${ }^{14}$ involved a mixture of cases in whom broad decompression and ensuing correction of deformation were carried out and cases in whom the decompression of short segmentation and fusion were performed. Therefore, there is a slight difference between this research and others in that correction of the deformity was made and the cases were considered together without focus on a correction of the deformation. Nonetheless, regardless of the intentions for deformity correction, their results are similar to these from the perspective that the deformity accelerates if there is lateral listhesis.

Although disc wedging has been presented as the etiology of degenerative lumbar scoliosis, there is no definitive research on the change in disc wedging after surgery. However, Ha et al. ${ }^{14}$ reported that although disc wedging improved after surgery and became even more severe during the follow-up, these were not statistically significant. In this study, the disc wedging angle continued to increase at all periods after surgery compared to that before surgery, and the difference was statistically significant up to 6 months after surgery $(p<0.05)$. However, it showed a slowing of rate of increase after 6 months following surgery, but these changes were not statistically significant $(\mathrm{p}>0.05)$. Therefore, more research with a sufficient follow-up observation and a greater number of cases will be necessary.

Regarding the complications, loosening of the pedicle screw was observed in 18 of the 47 patients. Twelve patients had loosening of the pedicle screw in the fusion at L5-S1 (4 patients with posterolateral fusion and 8 patients with posterior lumbar interbody fusion). In this study, the posterior approach was performed in all cases, and posterolateral fusion or posterior lumbar interbody fusion was also used. As a result, approximately $25 \%$ of patients showed nonunion at L5-S1.

Bridwell et al. ${ }^{15}$ reported that if fusion is performed up to L5 then degenerative changes can progress from L5-S1, and if fusion is performed up to $\mathrm{S} 1$, then there is an increase in nonunion of the lumbosacral region. Cho et al. ${ }^{5}$ reported similar results and degenerative changes of the lower segment, and that nonunion is more frequent in long segment fusion. This study examined the changes in the radiographical indices, including the progression of scoliotic curvature and changes in disc wedging, without investigating the changes in the upper or lower adjacent segments. However, 
if the clinical indices are to be researched together, this issue must be included because there is an increase in revision due to upper and lower adjacent segment problems. In addition, since there are definitive biomechanical differences between fusion up to L5 and fusion of the lumbosacral region ${ }^{16}$, a comparative study on the natural history of each would be an excellent research topic in the future.

\section{Conclusions}

Short segment decompression and fusion could correct scoliotic curvature, even though the degree was small, through appropriate compression following fixation with a pedicle screw and the correction of disc wedging by a cage in posterior lumbar interbody fusion. However, almost all cases showed allowable re-progression of the scoliotic curvature and disc wedging to different extents. Re-progression resumed approximately 6 to 9 months after the index surgery, and the extent of progression depended upon the degree of scoliotic angle before surgery.

In addition, a larger scoliotic angle before surgery and fusion up to the apical vertebra the accelerated scoliotic curvature rapidly, thereby highlighting precautions.

In general procedures of short segment decompression and fusion of degenerative lumbar scoliosis, it is difficult to state that cases without compressive fixation reflect the natural history after surgery because a certain extent of changes in scoliotic angle, i.e., small localized correction of curvature, is a process that always occurs. This study focused on changes in scoliotic curvature after short segment decompression and fusion, rather than the natural history of degenerative lumbar scoliosis.

However, this study had the limitation of a relatively short follow-up period as observed on the progression of degenerative lumbar scoliosis. Therefore, a re-evaluation should be performed after a long-term follow-up.

\section{REFERENCES}

1. Vanderpool DW, James JI, Wynne-Davies R: Scoliosis in the elderly. J Bone Joint Surg Am 1969; 51: 446-455.

2. Robin GC, Span Y, Steinberg R, Makin M, Menczel J: Scoliosis in the elderly: a follow-up study. Spine (Phila Pa 1976) 1982; 7: 355-359.

3. Kobayashi T, Atsuta Y, Takemitsu M, Matsuno T,
Takeda N: A prospective study of de novo scoliosis in a community based cohort. Spine (Phila Pa 1976) 2006; 31: 178-182.

4. Murata Y, Takahashi K, Hanaoka E, et al: Changes in scoliotic curvature and lordotic angle during the early phase of degenerative lumbar scoliosis. Spine (Phila Pa 1976) 2002; 27: 2268-2273.

5. Cho KJ, Suk SI, Park SR, et al: Short fusion versus long fusion for degenerative lumbar scoliosis. Eur Spine J 2008; 17: 650-656

6. Gupta MC: Degenerative scoliosis: options for surgical management. Orthop Clin North Am 2003; 34: 269-279.

7. Cho KJ, Park SL, Kim MG, et al: Proximal adjacent segment disease following posterior instrumentation and fusion for degenerative lumbar scoliosis. J Korean Orthop Assoc 2009; 44: 109-117.

8. Simmons ED: Surgical treatment of patients with lumbar spinal stenosis with associated scoliosis. Clin Orthop Relat Res 2001; (384): 45-53.

9. Kim YT, Lee CS, Kim JH, Kim JM, Park JH: Clinical features of degenerative scoliosis. J Korean Soc Spine Surg 2001; 8: 15-20.

10. Tribus CB: Degenerative lumbar scoliosis: evaluation and management. J Am Acad Orthop Surg 2003; 11: 174-183.

11. Bradford DS, Tay BK, Hu SS: Adult scoliosis: surgical indications, operative management, complications, and outcomes. Spine (Phila Pa 1976) 1999; 24: 2617-2629.

12. Wang MY, Green BA, Shah S, Vanni S, Levi AD: Complications associated with lumbar stenosis surgery in patients older than 75 years of age. Neurosurg Focus 2003; 14: e7.

13. Benz RJ, Ibrahim ZG, Afshar P, Garfin SR: Predicting complications in elderly patients undergoing lumbar decompression. Clin Orthop Relat Res 2001; (384): 116121.

14. Ha KY, Kim KW, Park SJ, Choi YS: Correction of curve and determination of fixation segment in degenerative lumbar scoliosis. J Korean Soc Spine Surg 2000; 7: 211-218.

15. Bridwell KH, Edwards CC 2nd, Lenke LG: The pros and cons to saving the L5-S1 motion segment in a long scoliosis fusion construct. Spine (Phila Pa 1976) 2003; 28: S234-S242.

16. Guan Y, Yoganandan N, Maiman DJ, Pintar FA: Internal and external responses of anterior lumbar/lumbosacral fusion: nonlinear finite element analysis. J Spinal Disord Tech 2008; 21: 299-304. 